\title{
Laser diagnostics for urea-water solution spray characterization
}

\author{
Eukasz Jan Kapusta ${ }^{1, *}$, and Andrzej Teodorczyk ${ }^{1}$ \\ ${ }^{1}$ Warsaw University of Technology, Faculty of Power and Aeronautical Engineering, ul. Nowowiejska 21/25, 00-665 Warszawa, Poland
}

\begin{abstract}
In this study, we summarize the laser techniques used for urea-water solution (UWS) spray characterization at the Institute of Heat Engineering (ITC), Faculty of Power and Aeronautical Engineering at Warsaw University of Technology. In presented studies several techniques for both, global and local spray characterization were used. Shadowgraphy-based long distance microscopy was used to visualize individual droplets and primary breakup. High speed imaging of Mie scattering (scattering on the gas-liquid interface) signal was used for global spray characterization. Combination of LIF (Laser Induced Fluorescence) and Mie scattering allowed to determine qualitative droplet size distribution across the whole spray cloud. The structured illumination technique used to modulate laser light sheet allowed to minimize the effects of multiple scattering in detection of Mie signal, what indicated huge potential of this technique in characterization of UWS sprays. The results presented here prove the importance of laser diagnostics in SCR systems development.
\end{abstract}

\section{Introduction}

Laser diagnostics for spray characterization has been well established in the area of fuel injection and mixture formation in both CI (Compression Ignition) and SI (Spark Ignition) engines. The application of sprays in CI engines due to implementation of SCR (Selective Catalytic Reduction) systems has extended. Moreover, due to development of close-coupled SCR system designs, and therefore decreased distance for water evaporation and urea decomposition the importance of spray in proper NOx reduction increases. NOx reduction efficiency strongly depends on the quality of mixing UWS with surrounding exhaust gases. UWS-surrounding gas mixing process is the main factor determining $\mathrm{NH} 3$ distribution before the inlet to the SCR catalyst where NOx reduction takes place.

The accurate measurement methods for fast and reliable UWS sprays characterization are of high importance in terms of spray pattern and injection process optimization. This is especially important for system optimization using CFD (Computational Fluid Mechanics) methods. It is of crucial importance to provide accurate input parameters such as droplet size distribution, spray angle, and spray pattern geometrical parameters.

In this paper, we summarize the laser techniques used to determine these parameters. The UWS spray characterization was done in the Laser Diagnostics Laboratory at Warsaw University of Technology. We used several techniques which allowed both, global and local spray characterization: shadowgraphy-based long distance microscopy, high speed imaging of Mie scattering (scattering on the gas-liquid interface),
LIF/Mie (Laser Induced Fluorescence together with Mie scattering).

Due to relatively large droplets and relatively dilute sprays generated by SCR system injectors the droplet diameters may be determined by direct visualization using high magnification optics. As shown by Grout et al. [1], even direct Mie scattering imaging can be used to determine the size of individual droplets. They used this approach to determine temporal change of the droplet size and calculate the evaporation rate according to Dsquare evaporation law. Commonly used method for individual droplets visualization is backlight imaging (shadowgraphy) [1,2]. This method coupled with long distance microscopy allows to visualize individual droplets of diameter lower than $10 \mu \mathrm{m}$ provided that the spray is not optically dense. This feature is important in terms of proper droplet size distribution determination especially when the range of droplet sizes is relatively wide. Therefore, in this study we used shadowgraphybased long distance microscopy. This technique allows also to precisely visualize primary breakup what was done in this study as well.

High speed imaging of Mie scattering signal is a convenient technique for fast global spray characterization. Simple global illumination (e.g. halogen lights) can be used for spray angle and spray tip penetration determination [3]. Its great advantage is that it can be used for determination of initial velocity of a liquid emerging the nozzle just after injector opening provided the frame rate is high.

LIF/Mie technique was used for qualitative analysis of droplet size distribution across the whole spray cloud. This technique was used for many years for gasoline spray characterization. It is based on simultaneous LIF and Mie signal measurements. Since under assumption

Corresponding author: lukasz.kapusta@itc.pw.edu.pl 
of spherical droplet LIF signal is proportional to the volume of the droplet and Mie signal to its surface the LIF/Mie ratio represents the SMD (Sauter Mean Diameter). In order to determine quantitative SMD distribution calibration factor for LIF/Mie ratio shall be first determined. The calibration procedure was not included in the study and the results presented here are intended to give qualitative information on the SMD distribution across the spray. As explained earlier, in order to determine SMD quantitatively shadowgraphybased long distance microscopy was used.

Recent findings of Berrocal [4] and Mishra [5] show that even in fairly dilute sprays effects of multiple scattering. Therefore, additionally Mie signals were captured using structured illumination. The results were compared with conventional Mie signals in order to conclude on the effects of multiple scattering on detected Mie signal and recognize the potential of this technique in LIF/Mie droplet sizing in the area of SCR sprays, what is planned to be done in near future.

\section{Experimental setup}

\subsection{Shadowgraphy-based long distance microscopy}

In all experiments the same injector was used (commercial 3-hole Bosch injector for SCR systems no. 0280158720 ). UWS in this study was replaced by pure water. Only in LIF/Mie droplet sizing study water was doped with eosin Y for the purpose of LIF signal generation. Injection pressure was of 5 bar. Water was injected into ambient conditions. The environment and water temperature was of $293 \mathrm{~K}$.

The setup for shadowgraphy included several components among which the most important was the Nd:YAG laser Quanta-Ray Pro-230. The 2nd harmonic of $532 \mathrm{~nm}$ wavelength was used to illuminate dye in the diffuser. The diffuser changed the wavelength to 574$580 \mathrm{~nm}$. The light emitted by the diffuser was used to illuminate the spray. The camera was equipped with long distance microscope, which was adjusted to visualize area of $88 \times 85 \mathrm{~mm}$ of the spray. The center of the image was located at $30 \mathrm{~mm}$ from the injector tip, in the axis of the injector. The schematic diagram of the experimental setup is shown in Fig. 1.

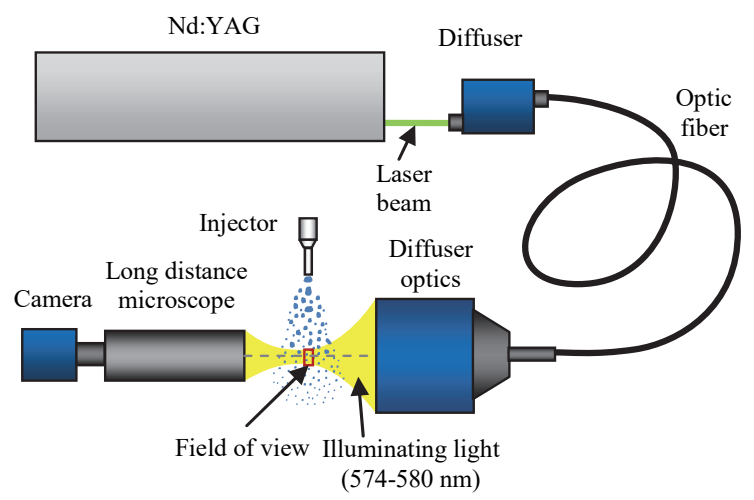

Fig. 1. Experimental setup for shadowgraphy [6].

\subsection{High speed imaging of Mie scattering}

The setup for high speed imaging of Mie scattering included the high speed camera and the light source. In this setup, global illumination was used. Two halogen lights of power $500 \mathrm{~W}$ each were used to illuminate the spray cloud. Photron SA1.1 camera with Nikon f 2.850 $\mathrm{mm}$ lens was used. The frame rate was of $10000 \mathrm{fps}$. The resolution was of $1024 \times 450$ pix. The setup is shown in Fig. 2.

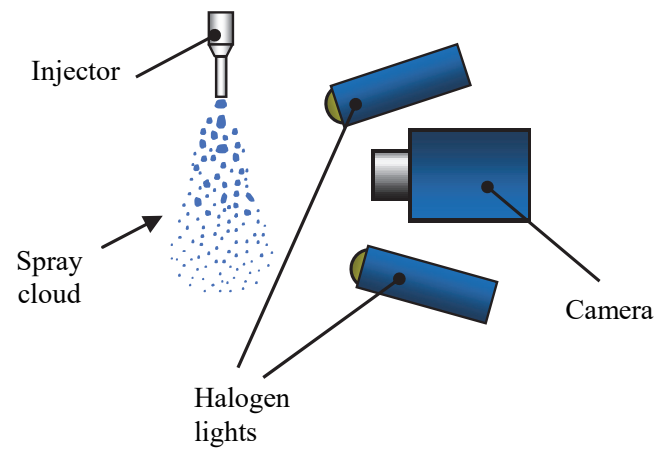

Fig. 2. Experimental setup for high speed imaging of Mie scattering.

\subsection{LIF/Mie droplet sizing}

In order to capture both signals LIF and Mie generated by the same droplets in the setup for LIF/Mie droplet sizing two cameras were used at the same time. Otherwise the number of images used for averaging would be significantly higher. The cameras were equipped with different filters to capture pure LIF and Mie signal. The cameras were located perpendicular to the light sheet - each camera was located at different side of the laser light sheet. As a light source second harmonic beam generated by Nd:YAG pulse laser was used. Note that injector used here was 3-hole injector and the spray cloud generated by the injector was formed by 3 single sprays. The light sheet was crossing one jet directly in its axis while going between the other two. The laser light sheet was of $60 \mathrm{~mm}$ wide and started $5 \mathrm{~mm}$ from injector outlet, since at the exit of the nozzle liquid ligaments and strongly deformed droplets are present. The experimental setup is shown in Fig. 3.

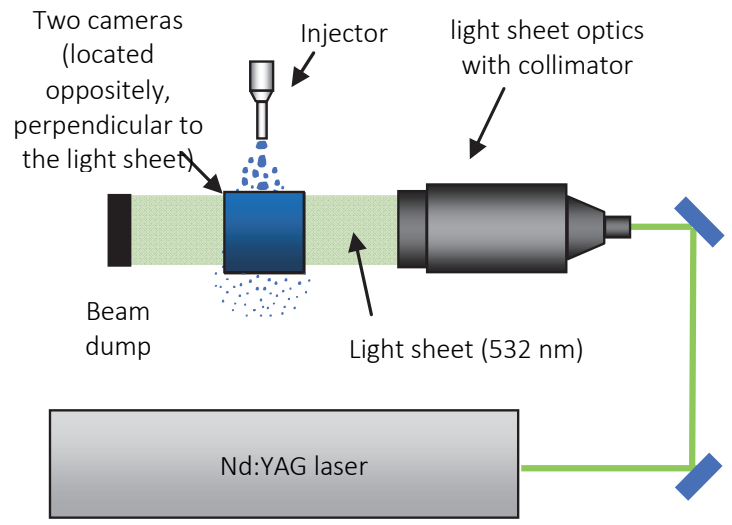

Fig. 3. Experimental setup for LIF/Mie measurements. 


\subsection{SLIPI}

The structured illumination technique referred to as SLIPI (Structured Illumination Planar Imaging) was developed for sprays by Berrocal et al. [7] and Kristenson et al. [8] and commercially implemented by LaVision [9]. The experimental setup including the SLIPI optics for structured light generation is shown in Fig. 4.

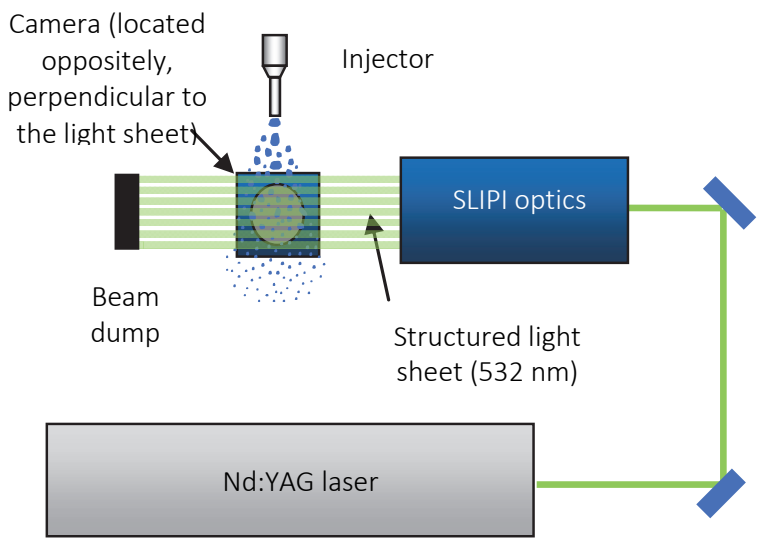

Fig. 4. Experimental setup for LIF/Mie measurement using structured illumination [10].

The modulated light sheet used in SLIPI visualization was entering the spray the same way as in LIF/Mie experiments, i.e. crossing one jet directly in its axis while going between the other two.

The SLIPI processing is performed by recording several modulated images where the modulation is vertically shifted between each recording [11]. The studies using structured illumination shown here were done only for Mie signal. The camera was located perpendicularly to the light sheet. As a light source again second harmonic beam generated by $\mathrm{Nd}$ :YAG pulse laser was used. The conventional images were reconstructed from three series of modulated images. The structured light sheet was created by SLIPI optics delivered by LaVision. The schematic setup of SLIPI optics is shown in Fig. 5.

In order to obtain LIF signal injected water was doped with Eosin Y.

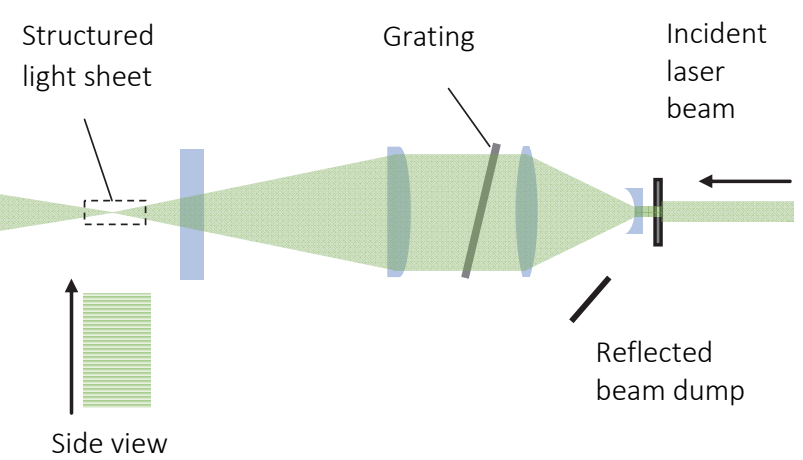

Fig. 5. Schematic setup of complete SLIPI optics - top view [10].

\section{Results}

\subsection{Shadowgraphy-based long distance microscopy}

Shadowgraphy-based long distance microscopy was used to visualize primary breakup (Fig. 6a) and determine droplet size distribution for the purpose of spray model calibration done in [12]. Due to frequency of the laser limited to $10 \mathrm{~Hz}$ only one image per injection was taken. The single image with indicated recognized particles is shown in Fig. $6 b$.

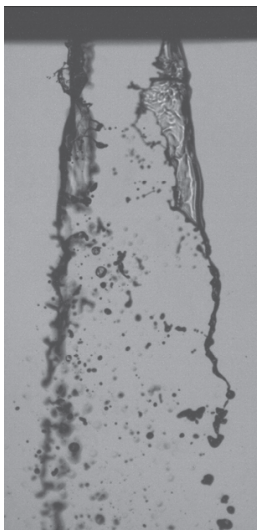

b)

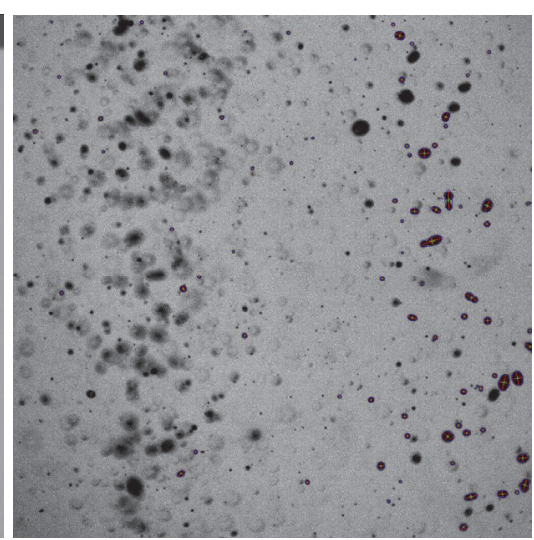

Fig. 6. Instantaneous shadowgraphy images; a) primary breakup (field of view: $39 \times 85 \mathrm{~mm}$ ), b) indicated recognized particles (field of view: $85 \times 88 \mathrm{~mm}$ ).

In order to perform accurate statistical analysis 100 injections were studied. At 100 images 6376 droplets in total were recognized. The detected droplet sizes were in the rage from $0 \mu \mathrm{m}$ to $300 \mu \mathrm{m}$. This range was divided into 30 subranges each of $10 \mu \mathrm{m}$ width. This data was used to construct droplet size distribution histogram (Fig. 7).

Basing on the acquired information on droplet sizes, SMD was calculated. The SMD was of $92.7 \mu \mathrm{m}$.

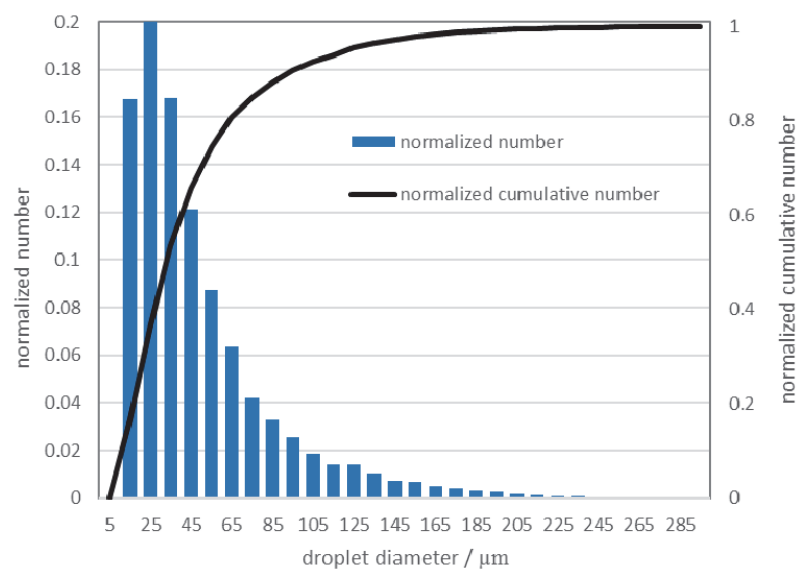

Fig. 7. Droplet size distribution. 


\subsection{High speed imaging of Mie scattering}

High speed imaging was used to determine spray penetration and initial liquid jet velocity in order to use the data for spray model calibration [12] as well. The single frames captured $1 \mathrm{~ms}, 2 \mathrm{~ms}, 3 \mathrm{~ms}$ and $7 \mathrm{~ms}$ after start of injection are shown in Fig. 8 respectively from left.
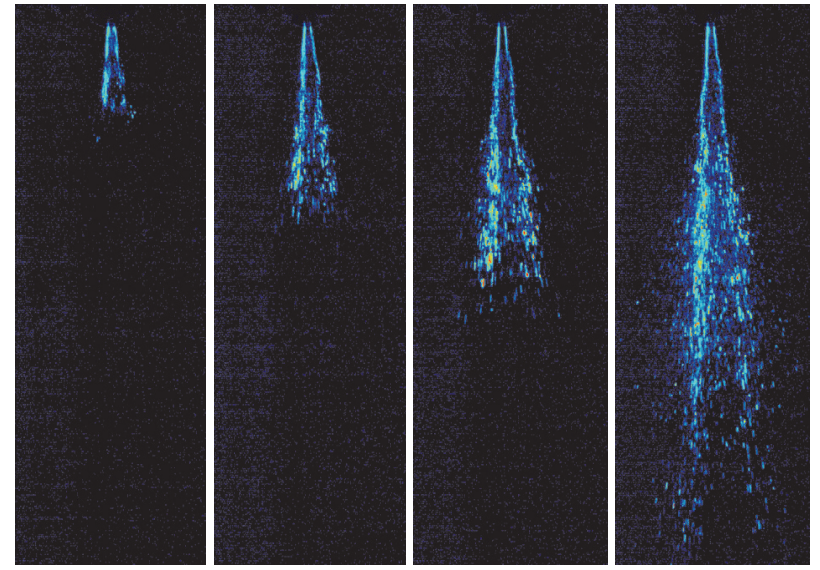

Fig. 8. Instantaneous Mie scattering high speed images $1 \mathrm{~ms}$, $2 \mathrm{~ms}, 3 \mathrm{~ms}$ and $7 \mathrm{~ms}$ after start of injection, respectively from left.

Basing on the acquired images the spray penetration was determined using LaVision DaVis version 8.2. The spray penetration evolution is shown in Fig. 9. The initial velocity of the liquid jet emerging the nozzle after the injector opening determined from first frames where jet occurred was of $24 \mathrm{~m} / \mathrm{s}$. The image where the spray was already developed was used to determine the spray visualization angle. Spray visualization angle determined from image captured $7 \mathrm{~ms}$ after start of injection was of $16.54 \mathrm{deg}$.

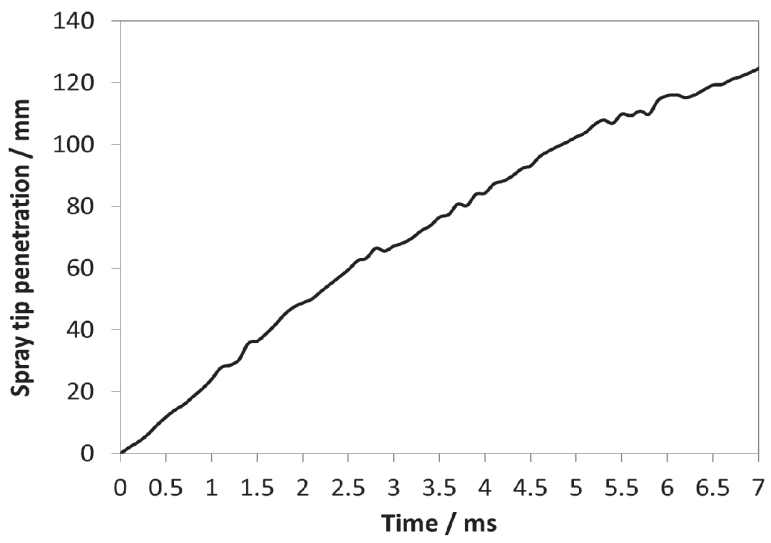

Fig. 9. Spray penetration evolution (adopted from [12]).

\subsection{LIF/Mie droplet sizing}

The procedure of image acquisition included obtaining 200 frames for each signal (LIF and Mie). Both signals were captured for the same laser pulse. Instantaneous LIF and Mie images for the same laser pulse are shown in Fig. 10. Note that the field of view at all single images presented in Figs 10-13 is of $60 \mathrm{~mm}$ high and $45 \mathrm{~mm}$ wide. Note that laser light sheet enters the spray from right on all LIF images and from left on all Mie images.

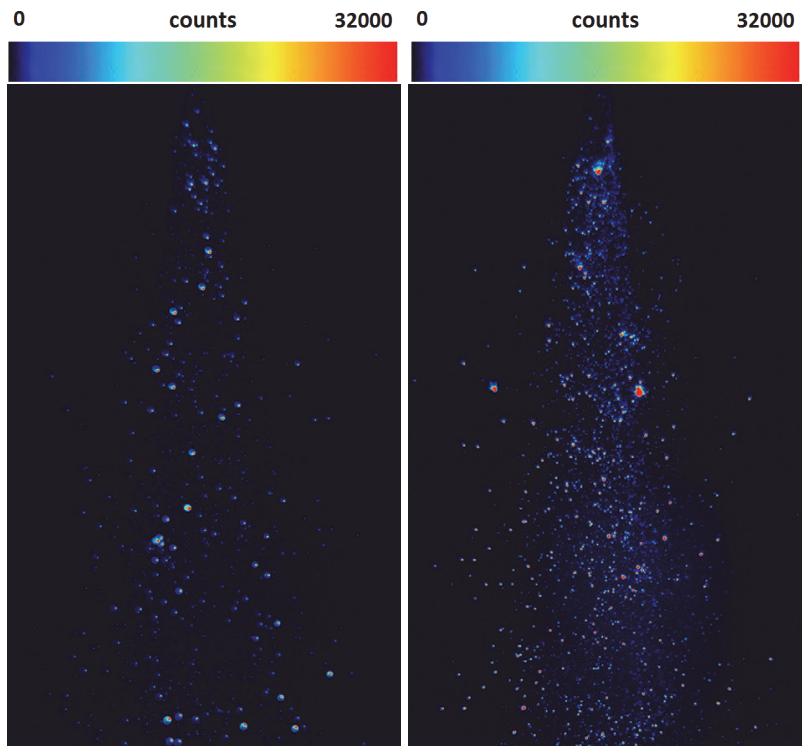

Fig. 10. Instantaneous LIF and Mie images, respectively from left.

Additionally to spray images the background image was recorded for each signal. The background images were recorded with laser pulse but without the spray. The instantaneous images shown in Fig. 10 were processed in order to subtract the background signal. Instantaneous LIF and Mie images for the same laser pulse after background subtraction are shown in Fig. 11. The 200 instantaneous images recorded for each signal after background subtraction were averaged. The averaged images of LIF and Mie signals are shown in Fig. 12. The averaged images shown in Fig. 12 were used to create LIF/Mie ratio image (shown in Fig. 13). Note that laser light sheet in Fig. 13 enters the spray from right.

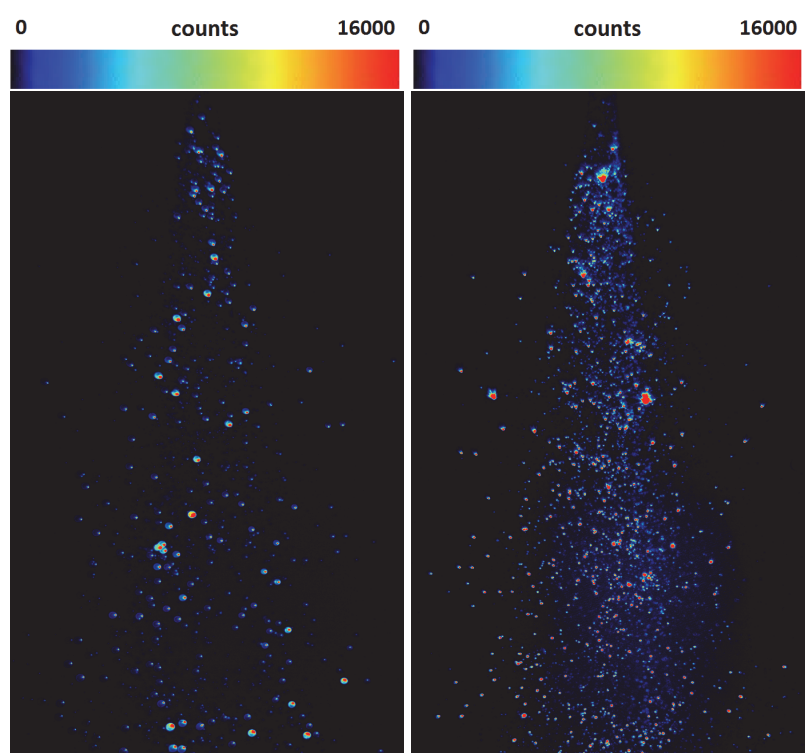

Fig. 11. Instantaneous LIF and Mie images after background subtraction, respectively from left. 

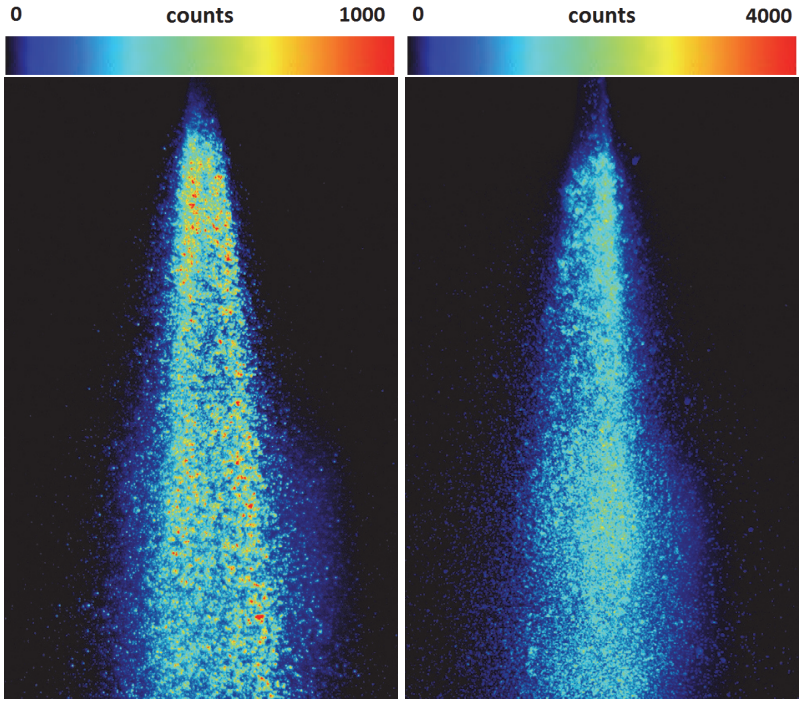

Fig. 12. Averaged LIF and Mie images, respectively from left.

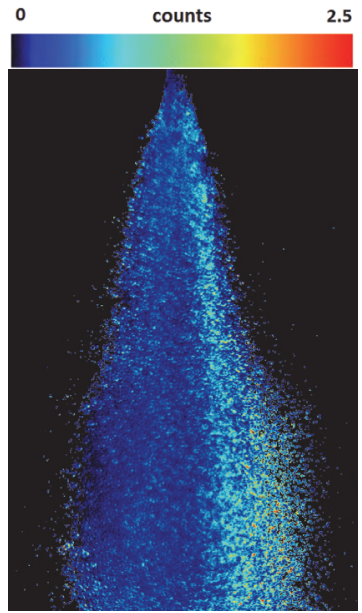

Fig. 13. LIF/Mie ratio.

LIF/Mie ratio in most of the visualized area remained unchanged, what suggests that SMD remains unchanged as well. One needs to be aware that at $90 \mathrm{deg}$ detection angle the scattered light intensity with the droplet diameter is irregular [13]. Therefore, LIF/Mie ratio shall be treated as qualitative indicator, not as direct SMD result. Slightly stronger signal can be observed along right nozzle axis (the spray emerging that nozzle was illuminated directly in the middle). This seems to be in accordance with the shadowgraphy results shown in Fig. $6 b$. There, between the jets only small number of very small droplets can be observed, while on the right side the main stream of large droplets is present. Note that the visualization depth of shadowgraphy is much higher than in case of light sheet imaging. Even though in Fig. $6 \mathrm{~b}$ large droplets are present also on right side of the image they are blurred. This indicates that they are located a bit closer or further than the focal point. Therefore, they are not detected by light sheet imaging. The other increase of LIF/Mie ratio can be observed at higher distance from the nozzle on the right side of the spray (Fig. 13), what suggests bigger droplets. This result is questionable since there is no reason to observe bigger droplets in that area. This might have come either from multiple scattering or other noise signal. Therefore the conventional imaging in case was compared with SLIPI imaging [10].

\subsection{SLIPI}

For the purpose of comparing conventional and SLIPI imaging, only Mie signal was used. The procedure of image acquisition included obtaining series of frames per one modulation phase. Full investigation into influence of number of frames on both SLIPI and conventional imaging results is shown in [10]. Here only results for case using 1000 frames per each phase are shown. Again, only one image per injection was captured. Recording series of 1000 images required 1000 separate injections. 1000 instantaneous images for each modulation phase were averaged. The average phase images are shown in Fig. 14.

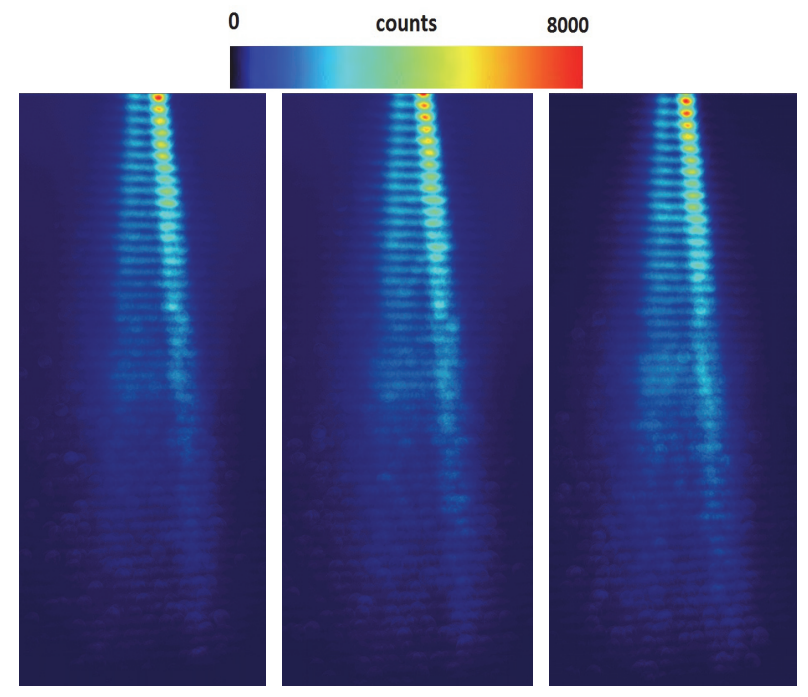

Fig. 14. Averaged Mie images for three modulation phases; the visualized area on single image is of $60 \mathrm{~mm}$ high and $22 \mathrm{~mm}$ wide.

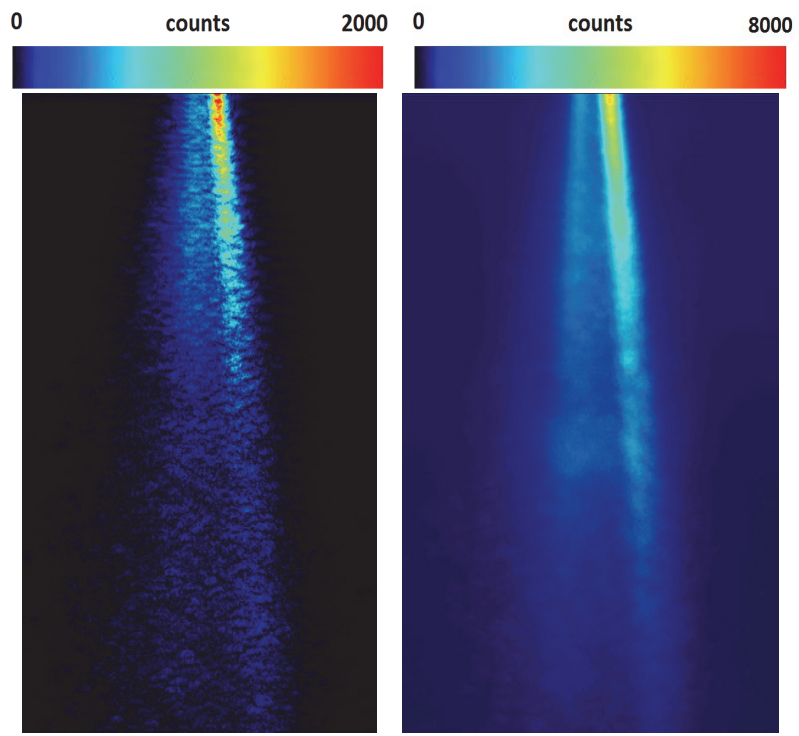

Fig. 15. SLIPI (left) and conventional (right) Mie scattering image [10]; the visualized area on single image is of $60 \mathrm{~mm}$ high and $35 \mathrm{~mm}$ wide. 
The averaged images were used to create SLIPI image and reconstruct conventional images. Both SLIPI and conventional images are shown in Fig. 15.

In general, averaged Mie signal determined using SLIPI and conventional processing were very similar. The SLIPI technique was however performing much better in areas between the single spray jets. In conventional image Mie signal between the spray jets was of similar intensity as in the background, especially at higher distances from the nozzle exit. The areas where droplets were observed in SLIPI image are clearly visible and can be easily distinguished from the background. The difference is much higher when SLIPI Mie signal (Fig. 15-left) is compared with Mie signal obtained using flat light sheet (Fig. 12-right). However, it needs to be noted that the number of frames used to create these images was different. The observed difference between SLIPI and conventional images indicates huge potential of SLIPI technique in droplet size distribution determination for SCR system applications by means of LIF/Mie method, what is planned to be done in near future.

Additionally, the obtained conventional and SLIPI images shown in Fig. 14 were used to determine the visualization angle. The visualization angle for that case was very similar for conventional and SLIPI imaging, $17.58 \mathrm{deg}$ and $17.7 \mathrm{deg}$ respectively.

\section{Conclusions}

The techniques used in the study were capable to deliver all the information on the sprays generated by the SCR system injector needed for their proper characterization. Such a wide information on spay parameters can be successfully used to adjust and calibrate CFD calculation setup for full SCR system investigations. Obtaining these parameters required several techniques which were based on the same laser system. Shadowgraphy-based long distance microscopy was used to visualize individual droplets and primary breakup. High speed imaging of Mie scattering allowed to determine global spray parameters, specifically spray tip penetration, and spray angle. Combination of LIF and Mie scattering allowed to determine qualitative 2D droplet size distribution across the whole spray cloud.

The studies done with structured illumination showed huge potential of SLIPI technique in droplet size distribution determination for SCR system applications by means of LIF/Mie method. This is planned to be done in near future.

It needs to be noted that the visualization angle determined using global illumination was different from the angle determined using light sheet imaging (both, SLIPI and conventional). For global illumination, the angle was of $16.54 \mathrm{deg}$ while for light sheet imaging was of $17.58 \mathrm{deg}$ and $17.7 \mathrm{deg}$ for conventional and SLIPI imaging, respectively. Imaging using global illumination is affected by the thickness of the spray cloud and therefore much stronger signal is detected in the center of the spray than in the peripheral region. Therefore, light sheet imaging result is more accurate.
The laser diagnostics provided all the data on SCR spray parameters critical for SCR systems development. In general, it may be concluded that the results presented in the study prove importance of laser imaging techniques in SCR systems development.

This work was supported by the European Smart Growth Operational Programme 2014-2020 through the project "Development of mixing and urea-water solution conversion unit in SCR systems in order to start production of exhaust system for compression ignition engine that meets the Euro 7 emission standards", grant number: POIR.04.01.04-000060/15-02.

\section{References}

1. S. Grout, J.B. Blaisot, K. Pajot, G. Osbat, Fuel 106, 166-177 (2013), DOI:10.1016/j.fuel.2012.09.022

2. L. Postrioti, G. Brizi, C. Ungaro, M. Mosser, F. Bianconi, Fuel 150, 548-557 (2015), DOI:10.1016/j.fuel.2015.02.067

3. I. Pielecha, International Journal of Automotive Technology 15, 47-55 (2014), DOI: 10.1007/s12239-014-0005-y

4. E. Berrocal, 18th Int Symp Appl Laser Imaging Tech to Fluid Mech (2016)

5. Y.N. Mishra, E. Kristensson, E. Berrocal, Opt $\begin{array}{llll}\text { Express 22, } & 4480 \quad \text { (2014), }\end{array}$ DOI:10.1364/OE.22.004480

6. L.J. Kapusta, P. Jaworski, A. Teodorczyk, J. Kowalski, Journal of KONES Powertrain Transp 22, 91-98 (2015), DOI:10.5604/12314005.1165976

7. E. Berrocal, E. Kristensson, M. Richter, M. Linne, M. Aldén, Opt Lett 16, 17870-17881 (2008), DOI:10.1364/OE.16.017870

8. E. Kristensson, E. Berrocal, M. Richter, S. Pettersson, M. Aldén, Opt Lett 33, 2752-2754 (2008), DOI:10.1364/OL.33.002752

9. P. Leick, R. Grzeszik, S. Arndt, S. Wissel, 24th Eur. Conf. Liq. At. Spray Syst. 1-10 (Estoril, 2011)

10. L.J. Kapusta, Proc. 8th Eur. Combust. Meet. 15411546 (Dubrovnik, 2017)

11. E. Berrocal, E. Kristensson, P. Hottenbach, M. Aldén, G. Grünefeld, Appl Phys B 109, 683-694 (2012), DOI:10.1007/s00340-012-5237-9

12. J. Bachanek, R. Rogóż, L.J. Kapusta, A. Teodorczyk, Proc. 8th Eur. Combust. Meet., 9961000 (Dubrovnik, 2017) 